\title{
Alteration to hippocampal shape in cannabis users with and without schizophrenia
}

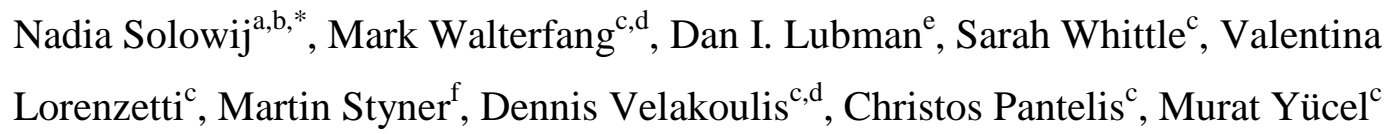

${ }^{a}$ School of Psychology, University of Wollongong, Wollongong NSW 2522, Australia

b Schizophrenia Research Institute, 405 Liverpool St Darlinghurst NSW 2010, Australia

${ }^{\mathrm{c}}$ Melbourne Neuropsychiatry Centre, University of Melbourne and Melbourne Health, Australia

d Neuropsychiatry Unit, Level 2, John Cade Building, Royal Melbourne Hospital 3050, Australia

e Turning Point Alcohol and Drug Centre, Eastern Health and Monash University, Australia

${ }^{\mathrm{f}}$ Department of Psychiatry and Department of Computer Science, University of North

Carolina, Chapel Hill NC, USA

* Corresponding author: School of Psychology, University of Wollongong, Wollongong NSW 2522, Australia. Tel.: +61 24221 3732; fax: +61 242214163.

E-mail address: nadia@uow.edu.au (N. Solowij) 


\begin{abstract}
Abnormalities in hippocampal morphology are characteristic of schizophrenia and have also been reported in chronic cannabis users. There is a paucity of research investigating potential additive effects of cannabis use on brain pathology associated with schizophrenia. In this study we performed hippocampal shape analysis in cannabis-using and non-using patients with schizophrenia, healthy cannabis users and healthy non-using controls. Hippocampal shape changes were observed in each group relative to controls, with the greatest degree of alterations (i.e., deflations across the hippocampus, and with an anterior predisposition), in cannabis-using schizophrenia patients. These alterations were associated with cannabis use patterns and psychotic symptoms.
\end{abstract}

Keywords: Cannabis, schizophrenia, hippocampus, shape analysis, neuroimaging 


\section{Introduction}

Cannabis use is highly comorbid with schizophrenia (Koskinen et al., 2010) and considered a component cause of the disorder (D’Souza et al., 2009; Murray et al., 2007). There is growing evidence that long-term or heavy cannabis use impacts upon brain structure and function, particularly in regions known to be affected in schizophrenia, such as the hippocampus (Ashtari et al., 2011; Lorenzetti et al., 2010; Solowij and Michie, 2007; Solowij et al., 2012). We have previously reported doserelated reductions in hippocampal volume in otherwise healthy chronic cannabis users that were associated with subclinical positive psychotic symptoms (Yücel et al., 2008), and were of a magnitude similar to that observed in schizophrenia (e.g. Velakoulis et al., 1999). A key question is whether effects associated with comorbid chronic cannabis use and schizophrenia exceed those associated with either condition occurring in isolation.

We sought to address this question by assessing hippocampal volume and performing hippocampal shape analysis to inform regional specificity in healthy cannabis users and in cannabis using and non-using patients with schizophrenia. In line with a recent study that reported greater hippocampal shape alterations in patients with schizophrenia and prior comorbid alcohol use disorders (Smith et al., 2011), we hypothesised that cannabis use would exert an additional effect on the hippocampal pathology typically observed in schizophrenia, particularly in the anterior hippocampus (Csernansky et al., 2002; Small et al., 2011; Tamminga et al., 2010). 


\section{Experimental/Materials and Methods}

\subsection{Participants, substance use and clinical measures}

Seventeen medicated patients with schizophrenia, 15 long-term heavy cannabis users (THC) and 16 healthy controls (CON), all right handed males, were recruited from the general community, by referral from psychiatrists or through the Australian Schizophrenia Research Bank register, and provided written informed consent. Eight of the patient group were long-term cannabis users (SZ+THC), with similar extensive levels of use as the healthy THC group (near daily for 10-32 years), while nine patients had never used cannabis regularly (SZ-THC). No participant had used any other illicit substance $>10$ times and alcohol use was limited to $<24$ standard drinks per week. All groups were matched on age (range 21-60 years), premorbid IQ (National Adult Reading Test) and education, but alcohol and tobacco use differed between groups. Demographic, clinical and substance use characteristics (elicited by structured interview) are provided in Table 1 (see also Solowij et al., 2011).

The Structured Clinical Interview for DSM-IV Axis I Disorders was used to exclude psychiatric disorders among healthy participants and to confirm a schizophrenia diagnosis in patients. Psychotic symptoms were assessed using the Scales for the Assessment of Positive and Negative Symptoms (SAPS and SANS; Andreasen, 1983). Healthy cannabis users had significantly higher SAPS $(z=3.57, p<.0005)$ and SANS $(z=3.66, p<.0005)$ scores than controls. The two patient groups (SZ+THC and SZTHC) did not differ in symptom scores (SAPS: $p>.19$; SANS: $p>.09$ ), which were higher than those observed in the healthy cannabis users (THC vs. SZ+THC, SAPS: $z=3.07, p=.002$; SANS: $z=3.39, p=.001$; THC vs. SZ-THC, SAPS: $z=1.88, p=.060$, 
SANS: $z=2.69, p=.007)$. All protocols were approved by university and regional health ethics committees.

\subsection{Neuroimaging procedures, measurements and statistical analyses}

MRI data were acquired from a 3-Tesla scanner using a volumetric SPGR sequence with 180 contiguous coronal slices (TE, 2.9ms; TR, 6.4ms; flip-angle, $8^{\circ}$; matrix-size, 256x256; $1 \mathrm{~mm}^{3}$ voxels).

Hippocampal volumes were measured using established protocols (Velakoulis et al., 1999) and delineated by a trained rater blind to group information (see Yücel et al, 2008). Left and right hippocampal volumes were compared between groups by ANOVA. Shape analysis was undertaken in a semi-automated fashion using the University of North Carolina shape analysis toolkit - spherical harmonic shape description (SPHARM-PDM; Brechbuhler et al., 1995); a detailed description of the methodology is available in Styner et al. (2004; 2006). We utilized MANCOVA within SPHARM-PDM to control for alcohol and tobacco use in between-group analyses, while controlling for multiple comparisons using the false discovery rate (FDR) correction procedure (Paniagua et al., 2009; Pantazis et al., 2004; Styner et al., 2004), and the toolkit's correlational analysis to calculate Spearman's correlations with cannabis use parameters and psychotic symptoms. 


\section{Results}

\subsection{Hippocampal volume}

Neither tobacco nor alcohol use correlated with hippocampal volumes in the entire sample (cigarettes/day: left: -.09, $p=.66$; right: -.13, $p=.52$; standard drinks/day: left: $.18, p=.29$; right: $-.14, p=.42$ ) or in any subgroup, and were therefore not included as covariates. The overall difference between the 4 groups was significant for both left and right hippocampus (Table 1) with the THC group having smaller hippocampi bilaterally than CON (left: $\mathrm{F}_{1,29}=9.25, p=.005$; right: $\mathrm{F}_{1,29}=11.05, p=.002$ ). The THC group did not differ from either of the schizophrenia groups (SZ+THC: left $p=.18$, right $p=.15$; SZ-THC: left $p=.11$, right $p=.62$ ). The SZ+THC group did not differ significantly from controls (left $p=.26$, right $p=.25$ ), whereas SZ-THC had smaller right $\left(\mathrm{F}_{1,23}=5.33, p=.03\right)$ but not left $(p=.23)$ hippocampi than CON. Combining the two schizophrenia groups confirmed a significant difference from CON for the right $\left(\mathrm{F}_{1,31}=4.88, p=.035\right)$ but not left $(p=.14)$ hippocampus. SZ+THC and SZ-THC did not differ in volume (left $p=.96$, right $p=.39$ ).

\subsection{Between-group shape comparisons}

Fig. 1 shows the raw and FDR-corrected $p$-value and difference maps from betweengroup comparisons of hippocampal shape, with each clinical group compared against CON. Regional shape alterations were observed in each group, but only the large reductions (areas of deflation up to $4 \mathrm{~mm}$ ) in the left hippocampus of the SZ+THC group survived the conservative FDR correction. The overall shape change in the left hippocampus was significant between SZ+THC and CON ( $p=.003)$, falling just short of significance on the right ( $p=.058$ ) (with areas of deflation up to $2 \mathrm{~mm}$ ). SZ-THC 
showed shape alterations in regions of the head and body of the hippocampus, with areas of deflation of approximately $2 \mathrm{~mm}$ in the left and $1.5 \mathrm{~mm}$ in the right hippocampus. The overall shape change across the surface showed trend level significance for SZ-THC compared to CON ( $p=.056)$ in the left hippocampus, but not in the right $(p=.24)$. Similarly, THC showed bilateral differences compared to CON, with larger regions of deflation in the right hippocampus, and of a similar magnitude to that observed in SZ-THC. There was a significant overall shape change for THC compared to CON in the right hippocampus $(p=.045)$, with a trend in the left hippocampus ( $p=.085)$. The same pattern of results was obtained after covarying for alcohol and tobacco use.

\subsection{Correlational shape analyses}

The cumulative dose of exposure to cannabis, amount and frequency of use in the past month, and duration of use, all showed significant negative correlation with hippocampal shape in the cannabis-using group as a whole (THC and SZ+THC) (examples shown in Fig. 2), suggesting greater regional deflation with greater exposure to cannabis. Duration of illness in the schizophrenia group as a whole (SZ+THC and SZ-THC) correlated with the shape of some regions of the hippocampal head, being reduced with increasing years of illness. SAPS and SANS symptom scores correlated with hippocampal shape in the THC, SZ+THC and SZTHC groups, most prominently in SZ+THC, with both positive and negative symptom scores showing a strong correlation with widespread hippocampal deflation bilaterally (Fig. 3). However, none of these correlations survived FDR correction, likely due to the small sample size. 


\section{Discussion}

Hippocampal pathophysiology is evident in a diverse range of disorders that appear to differentially target distinct subregions of the hippocampus (Small et al., 2011). Despite our limited sample size, shape analysis determined additive effects of cannabis to the hippocampal pathology evident in schizophrenia, when significant volumetric differences were not observed between patients who used cannabis and those that did not. The regional specificity of changes in the shape of the hippocampus in SZ+THC, most significant in the tip of the tail, head and midway down the body, supports the sensitivity of shape analysis to detecting alterations in the absence of a volume difference. The global shape change in the THC group, on the other hand, suggests more diffuse alterations that parallel the overall volume reduction observed. Cannabis exposure in both groups was associated with deflations in anterior and medial areas that may correspond to the dentate gyrus and CA3/4 regions. We also observed strong associations between hippocampal regional deflations and psychotic symptoms in the cannabis-using schizophrenia patients, findings that require replication with a larger sample.

While the mechanism underlying hippocampal shape changes is not known, the altered regions are dense with cannabinoid receptors (Glass et al., 1997), which may be dysfunctional in the pathology of schizophrenia and as a result of long-term exposure to cannabis (Villares, 2007). Our findings support an interpretation of dosedependent neurotoxic effects, broadly across the hippocampus, as evidenced also by animal studies (see Solowij et al., 2012). The combined effects of comorbid chronic cannabis use and schizophrenia appear to exceed those of either condition occurring 
in isolation, consistent with other recent studies (Habets et al., 2011; Rais et al., 2008; Solowij et al., 2011). Future studies with larger samples could examine further gender effects and the functional, clinical and neuropsychological correlates of this neuropathology.

\section{References}

Andreasen, N.C., 1983. The Scales for the Assessment of Positive (SAPS) and Negative (SANS) Symptoms. University of Iowa: Iowa City.

Ashtari, M., Avants, B., Cyckowski, L., Cervellione, K.L., Roofeh, D., Cook, P., et al., 2011. Medial temporal structures and memory functions in adolescents with heavy cannabis use. J. Psychiatr. Res. 45 (8) 1055-1066.

Brechbuhler, C., Gerig, G., Kubler, O., 1995. Parameterization of closed surfaces for 3-D shape description. Comput. Vis. Image Und. 61 (2) 154-170.

Csernansky, J.G., Wang, L., Jones, D., Rastogi-Cruz, D., Posener, J.A., Heydebrand, G., et al., 2002. Hippocampal deformities in schizophrenia characterized by high dimensional brain mapping. Am J Psychiat. 159 (12), 2000-2006.

D’Souza, D.C., Sewell, R.A., Ranganathan, M., 2009. Cannabis and psychosis/schizophrenia: human studies. Eur. Arch. Psy. Clin. N. 259 (7) 413431.

Glass, M., Dragunow, M., Faull, R.L.M., 1997. Cannabinoid receptors in the human brain: A detailed anatomical and quantitative autoradiographic study in the fetal, neonatal and adult human brain. Neuroscience. 77 (2), 299-318.

Habets, P., Marcelis, M., Gronenschild, E., Drukker, M., van Os, J., 2010. Reduced cortical thickness as an outcome of differential sensitivity to environmental risks in schizophrenia. Biol. Psychiat. 69 (5) 487-494.

Koskinen, J., Löhönen, J., Koponen, H., Isohanni, M., Miettunen, J., 2010. Rate of cannabis use disorders in clinical samples of patients with schizophrenia: a meta-analysis. Schizophrenia Bull. 36 (6) 1115-1130. 
Lorenzetti, V., Lubman, D.I., Whittle, S., Solowij, N., Yücel, M., 2010. Structural MRI findings in long-term cannabis users: What do we know? Subst. Use Misuse. 45 1787-1808.

Murray, R.M., Morrison, P.D., Henquet, C., Di Forti, M., 2007. Cannabis, the mind and society: the hash realities. Nat. Rev. Neurosci. 8 (11) 885-895.

Paniagua, B., Styner, M., Macenko, M., Pantazis, D., Niethammer, M., 2009. Local shape analysis using MANCOVA. Insight. 1-21: http://hdl.handle.net/10380/3124.

Pantazis, D., Leahy, R., Nichols, T., Styner, M., 2004. Statistical surface-based morphometry using a non-parametric approach. $2^{\text {nd }}$ IEEE Int. Symp. Biomed. Imaging: Macro to Nano, Vol 1 \& 2, 1283-1286.

Rais, M., Cahn, W., Van Haren, N., Schnack, H., Caspers, E., Hulshoff Pol, H., et al., 2008. Excessive brain volume loss over time in cannabis-using first episode schizophrenia patients. Am J Psychiat. 165 (4) 490-496.

Small, S.A., Schobel, S.A., Buxton, R.B., Witter, M.P., Barnes, C.A., 2011. A pathophysiological framework of hippocampal dysfunction in ageing and disease. Nat. Rev. Neurosci. 12 (10) 585-601.

Smith, M.J., Wang, L., Cronenwett, W., Goldman, M.B., Mamah, D., Barch, D., et al., 2011. Alcohol use disorders contribute to hippocampal and subcortical shape differences in schizophrenia. Schizophr. Res. 131 (1-3) 174-183.

Solowij, N., Michie, P.T., 2007. Cannabis and cognitive dysfunction: Parallels with endophenotypes of schizophrenia? J. Psychiatr. Neurosci. 32 (1) 30-52.

Solowij, N., Yücel, M., Lorenzetti, V., Lubman, D.I., 2012. Does cannabis cause lasting brain damage? In Castle, D., Murray, R. and D’Souza, D.C. (Eds) Marijuana and Madness (2nd ed.), (pp. 103-113). Cambridge: Cambridge University Press.

Solowij, N., Yücel, M., Respondek, C., Whittle, S., Lindsay, E., Pantelis, C., et al., 2011. Cerebellar white matter changes in cannabis users with and without schizophrenia. Psychol. Med. 41 (11) 2349-2359.

Styner, M., Lieberman, J., Pantazis, D., Gerig, G., 2004. Boundary and medial shape analysis of the hippocampus in schizophrenia. Med. Image Anal. 8 (3) 197-203.

Styner, M., Oguz, I., Xu, S., Brechbuhler, C., Pantazis, D., Levitt, J., et al., 2006. Framework for the statistical shape analysis of brain structures using SPHARMPDM. Insight: http://hdl.handle.net/1926/215. 
Tamminga, C.A., Stan, A.D., Wagner, A.D., 2010. The hippocampal formation in schizophrenia. Am. J. Psychiatr. 167 (10) 1178-1193.

Velakoulis, D., Pantelis, C., McGorry, P.D., Dudgeon, P., Brewer, W., Cook, M., et al., 1999. Hippocampal volume in first-episode psychoses and chronic schizophrenia: a high-resolution magnetic resonance imaging study. Arch. Gen. Psychiatr. 56 (2) 133-141.

Villares, J., 2007. Chronic use of marijuana decreases cannabinoid receptor binding and mRNA expression in the human brain. Neuroscience. 145 323-334.

Yücel, M., Solowij, N., Respondek, C., Whittle, S., Fornito, A., Pantelis, C., et al., 2008. Regional brain abnormalities associated with long-term heavy cannabis use. Arch. Gen. Psychiatr. 65 (6) 694-701. 


\section{Figure legends}

Fig. 1. Shape statistical analysis significance maps showing comparisons between schizophrenia patients without cannabis use (SZ+THC) versus healthy controls (CON) (top), schizophrenia patients with cannabis use (SZ-THC) versus CON (middle) and healthy cannabis users (THC) versus CON (bottom). Left hippocampus is depicted on the left and right hippocampus on the right, displaying superior and inferior views. For each view, raw $p$-value maps, FDR-corrected $p$-value maps, and mean difference magnitude displacement maps are shown (the latter visualising group test local effect size by displaying the magnitude of deflation (in $\mathrm{mm}$ ) between the same point on the mean surface of each group (Styner et al., 2006)).

Fig. 2. Correlations in the entire cannabis-smoking group (THC and SZ+THC) between hippocampal shape and cumulative exposure to cannabis over the past 10 years (top) and frequency of cannabis use in the past month (bottom), with left hippocampus on the left and right hippocampus on the right, displaying superior and inferior views. Raw p-value maps and Spearman's rho-value maps are shown. The significance of these correlations did not survive FDR correction (FDR-corrected $p$ value maps not shown).

Fig. 3. Correlations in the cannabis-using schizophrenia group (SZ+THC) between hippocampal shape and positive symptoms (SAPS score; top) and negative symptoms (SANS score; bottom), with left hippocampus on the left and right hippocampus on the right, displaying superior and inferior views. Raw p-value maps and Spearman's 
rho-value maps are shown. The significance of these correlations did not survive FDR correction (FDR-corrected $p$-value maps not shown). 
Table 1: Demographic, clinical, drug use and MRI volumetric measures: mean (SD) or median [range].

\begin{tabular}{|c|c|c|c|c|c|}
\hline & $\begin{array}{l}\text { THC } \\
\mathrm{n}=15\end{array}$ & $\begin{array}{l}\mathrm{CON} \\
\mathrm{n}=16\end{array}$ & $\begin{array}{c}\mathrm{SZ}+\mathrm{THC} \\
\mathrm{n}=8\end{array}$ & $\begin{array}{c}\text { SZ-THC } \\
n=9\end{array}$ & p-value ${ }^{a}$ \\
\hline Age & 39.8 (8.9) & $36.4(9.8)$ & 37.5 (6.6) & $44.1(8.6)$ & 0.21 \\
\hline IQ & $109.2(6.3)$ & $113.9(8.1)$ & $110.6(9.2)$ & 105.5 (11.8) & 0.14 \\
\hline $\begin{array}{l}\text { Years of } \\
\text { education }\end{array}$ & 13.5 (3.2) & $14.8(3.7)$ & $13.4(3.0)$ & 14.9 (3.8) & 0.58 \\
\hline $\begin{array}{l}\text { SAPS composite } \\
\text { score }\end{array}$ & $6.0[0-28]$ & $0.0[0-4]$ & 30.0 [12-43] & 16.0 [3-43] & $<0.001$ \\
\hline $\begin{array}{l}\text { SANS composite } \\
\text { score }\end{array}$ & $12.0[0-25]$ & $1.5[0-4]$ & 31.0 [16-43] & 24.0 [12-35] & $<0.001$ \\
\hline $\begin{array}{l}\text { Duration of } \\
\text { illness }\end{array}$ & - & - & $14.1(5.9)$ & $23.6(11.2)$ & 0.049 \\
\hline $\begin{array}{l}\text { Age at diagnosis } \\
\text { Cannabis: }\end{array}$ & - & - & 20.5 [17-37] & 20.0 [16-27] & 0.56 \\
\hline $\begin{array}{l}\text { Years of regular } \\
\text { Use }^{\mathrm{b}}\end{array}$ & $\begin{array}{c}19.7(7.3), \\
\text { range } 10-32\end{array}$ & - & $\begin{array}{c}17.9(6.5), \\
\text { range } 11-29\end{array}$ & - & 0.57 \\
\hline $\begin{array}{l}\text { Age started } \\
\text { regular use }^{\mathrm{b}}\end{array}$ & $\begin{array}{c}20.1(5.4), \\
\text { range } 12-34\end{array}$ & - & $\begin{array}{c}19.6(6.2), \\
\text { range } 13-29\end{array}$ & - & 0.84 \\
\hline $\begin{array}{l}\text { Current use } \\
\text { (days/mnth) }^{\mathrm{c}}\end{array}$ & $28(4.6)$ & - & $25(8.1)$ & - & 0.34 \\
\hline $\begin{array}{l}\text { Current use } \\
\text { (cones/mnth) }^{\mathrm{c}}\end{array}$ & $636(565)$ & - & $644(344)$ & - & 0.97 \\
\hline $\begin{array}{l}\text { Cumulative } \\
\text { exposure (past } \\
10 \mathrm{yrs})^{\mathrm{c}}\end{array}$ & $\begin{array}{c}77816 \\
(66542)\end{array}$ & - & $\begin{array}{c}62925 \\
(25756)\end{array}$ & - & 0.55 \\
\hline $\begin{array}{l}\text { Alcohol (stand. } \\
\text { drinks/week) }\end{array}$ & 7.0 [0-24] & $4.0[0-16]$ & $7.0[0-21]$ & $0[0-10]$ & 0.028 \\
\hline $\begin{array}{l}\text { Tobacco } \\
\text { (cigarettes/day) }\end{array}$ & 20 [1-35] & 0 [0-14] & 20 [0-35] & 7 [0-35] & $<0.001$ \\
\hline $\begin{array}{l}\text { Intracranial } \\
\text { cavity }^{\mathrm{d}}\end{array}$ & $\begin{array}{c}1546237 \\
(94018)\end{array}$ & $\begin{array}{l}1607590 \\
(126386)\end{array}$ & $\begin{array}{l}1539072 \\
(157846)\end{array}$ & $\begin{array}{l}1486687 \\
(164022)\end{array}$ & 0.17 \\
\hline Whole brain & 1310780 & 1374123 & 1293879 & 1239308 & 0.063 \\
\hline
\end{tabular}




\begin{tabular}{|c|c|c|c|c|c|}
\hline volume $^{\mathrm{c}}$ & (90 779) & (105 673) & (145 199) & (155 894) & \\
\hline Left hippocampal & 2849 & 3240 & 3036 & 3044 & 0.03 \\
\hline volume $^{\mathrm{c}}$ & $(270)$ & $(423)$ & $(374)$ & $(300)$ & \\
\hline Right & 2949 & 3348 & 3144 & 3003 & 0.01 \\
\hline hippocampal & $(244)$ & $(400)$ & (383) & $(264)$ & \\
\hline volume $e^{\mathrm{c}}$ & & & & & \\
\hline
\end{tabular}

a Difference between the 4 groups or 2 groups depicted in each row from ANOVA, Kruskal-Wallis or Mann-Whitney tests.

${ }^{\mathrm{b}}$ Regular use was defined as at least twice a month

c Cannabis users had used at this level for the majority of their drug-using careers; a 'cone' is the small funnel into which cannabis is packed to consume through a water pipe in a single inhalation. Without the loss of sidestream smoke, the quantity of THC delivered by this method is estimated as equating three cones to one cigarette-sized joint. Thus, the cannabis users with and without schizophrenia smoked the equivalent of 213 joints per month, or approximately 7 joints per day. Estimates of lifetime dose beyond 10 years in these very long-term users became skewed and unreliable, hence the 10-year estimate was used in correlational analyses.

${ }^{\mathrm{d}}$ Measures of brain volumes corrected for intracranial cavity are in units of $\mathrm{mm}^{3}$. 


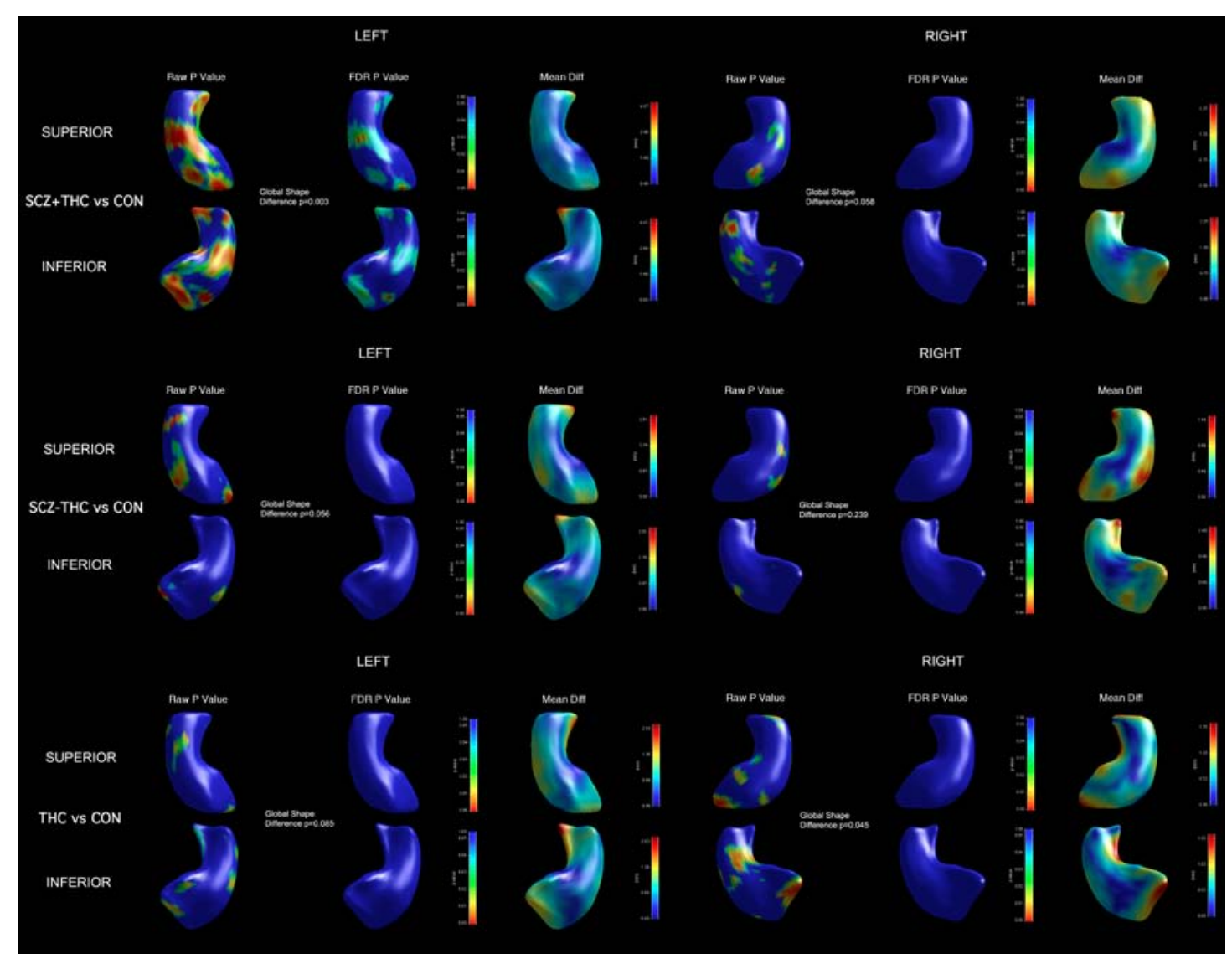

Fig. 1. Shape statistical analysis significance maps showing comparisons between schizophrenia patients without cannabis use (SZ-THC) versus healthy controls (CON) (top), healthy cannabis users (THC) versus CON (middle) and schizophrenia patients with cannabis use (SZ+THC) versus CON (bottom). Left hippocampus is depicted on the left and right hippocampus on the right, displaying superior and inferior views. For each view, raw p-value maps, FDR-corrected p-value maps, and mean difference magnitude displacement maps are shown (the latter visualising group test local effect size by displaying the local magnitude of deflation (in mm) on the overall mean surface (Styner et al., 2006)). 


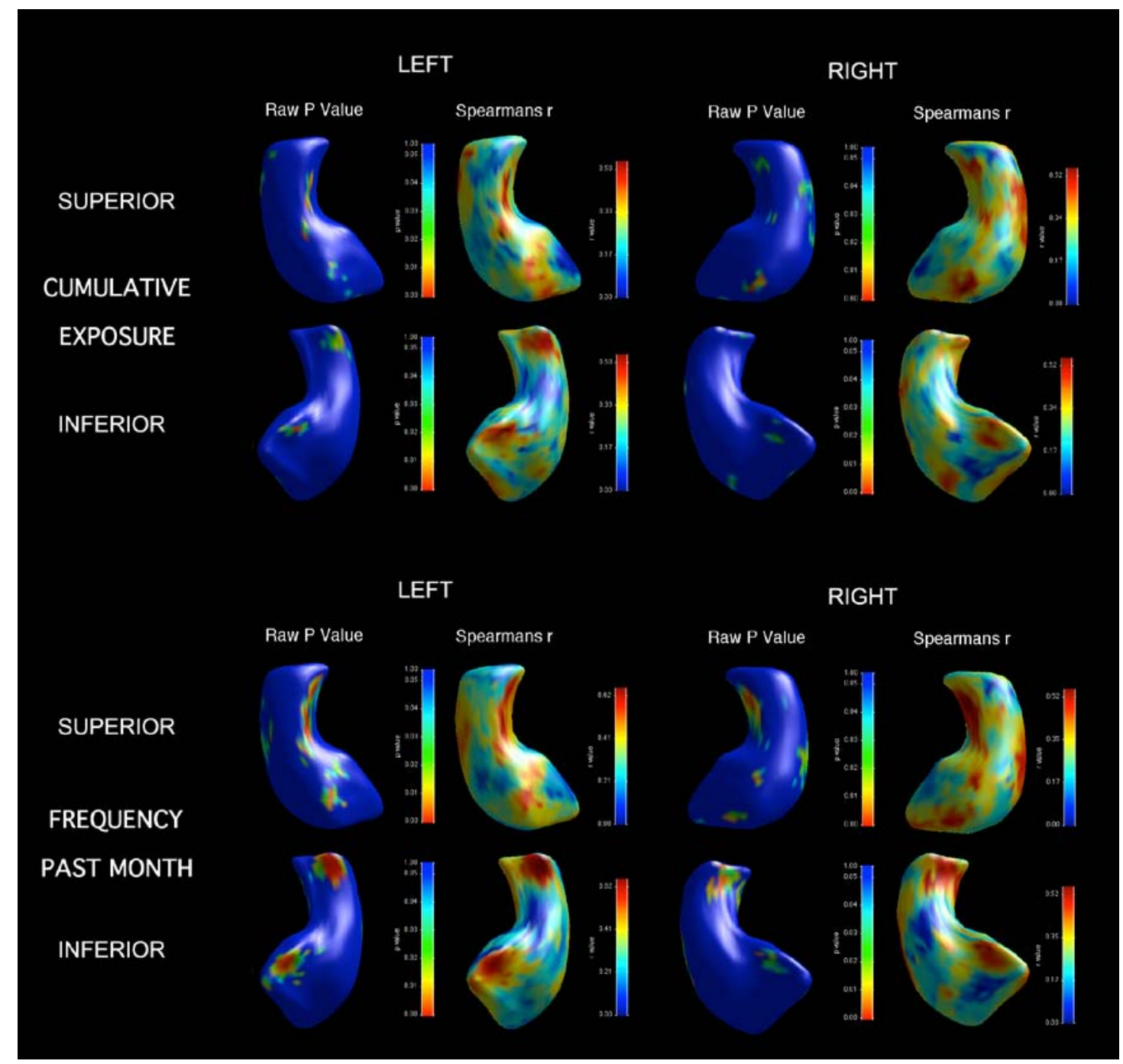

Fig. 2. Correlations in the entire cannabis-smoking group (THC and SZ+THC) between hippocampal shape and cumulative exposure to cannabis over the past 10 years (top) and frequency of cannabis use in the past month (bottom), with left hippocampus on the left and right hippocampus on the right, displaying superior and inferior views. Raw $p$-value maps and Spearman's rho-value maps are shown. The significance of these correlations did not survive FDR correction (FDR-corrected $p$-value maps not shown). 


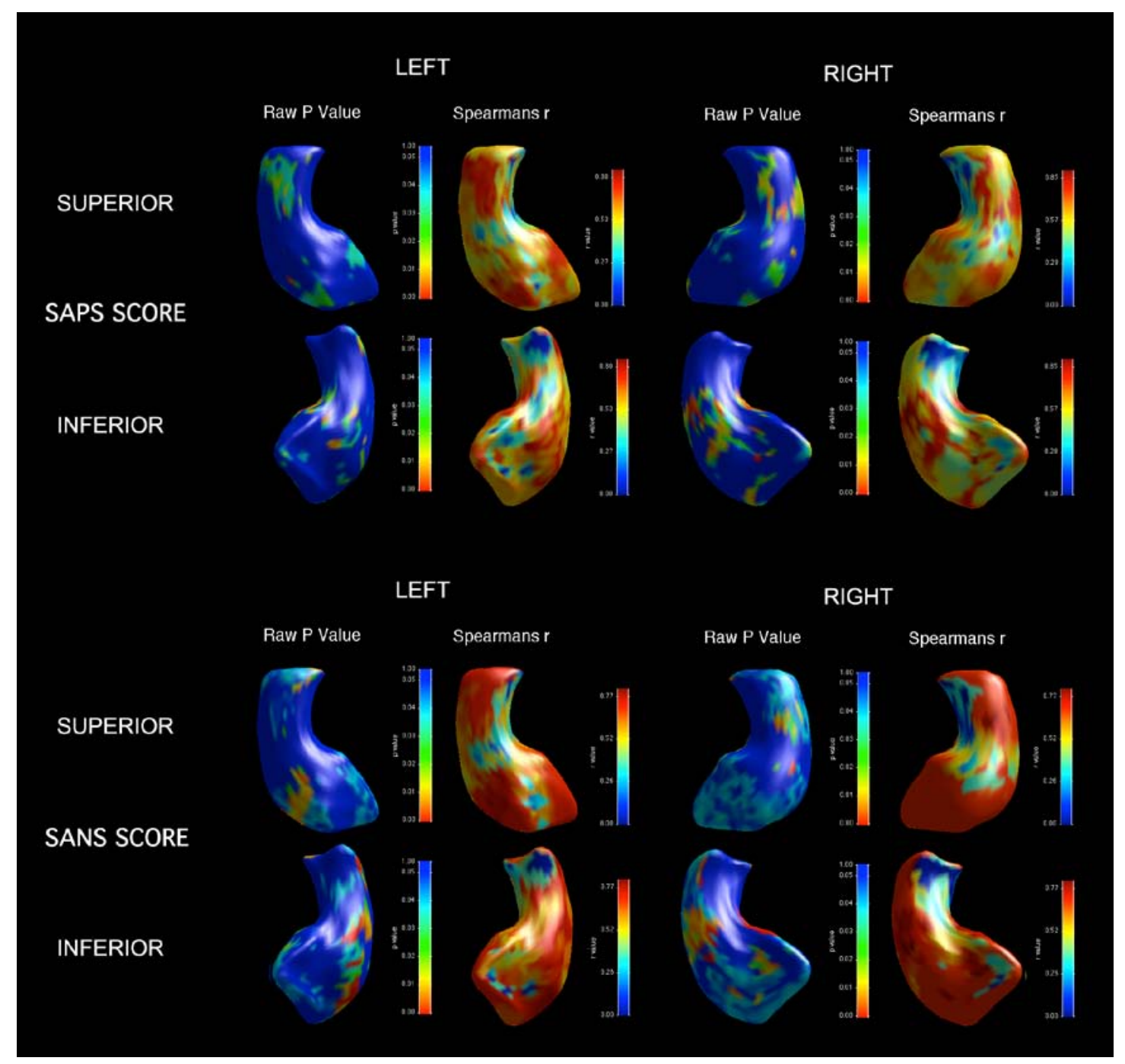

Fig. 3. Correlations in the cannabis-using schizophrenia group (SZ+THC) between hippocampal shape and positive symptoms (SAPS score; top) and negative symptoms (SANS score; bottom), with left hippocampus on the left and right hippocampus on the right, displaying superior and inferior views. Raw $p$-value maps and Spearman's rho-value maps are shown. The significance of these correlations did not survive FDR correction (FDR-corrected $p$-value maps not shown). 